\title{
Téoros
}

Revue de recherche en tourisme

\section{Les habitudes de voyages et de loisirs des producteurs agricoles québécois}

\section{Pierre Filiatrault et Hans Laroche}

Volume 4, numéro 2, juillet 1985

Les clientèles touristiques : un tour d'horizon

URI : https://id.erudit.org/iderudit/1080629ar

DOI : https://doi.org/10.7202/1080629ar

Aller au sommaire du numéro

Éditeur(s)

Université du Québec à Montréal

ISSN

0712-8657 (imprimé)

1923-2705 (numérique)

Découvrir la revue

Citer cet article

Filiatrault, P. \& Laroche, H. (1985). Les habitudes de voyages et de loisirs des producteurs agricoles québécois. Téoros, 4(2), 26-28.

https://doi.org/10.7202/1080629ar d'utilisation que vous pouvez consulter en ligne. 


\title{
Les habitudes de voyages et de loisirs des producteurs agricoles québécois
}

\author{
par Pierre Filiatrault et Hans Laroche*
}

Relativement peu de recherches ont été effectuées sur les activités de vacances et de loisirs des producteurs agricoles québécois. Quelle est la propension des producteurs agricoles à voyager durant leurs vacances? Quelles sont leurs destinations? Quelles sont leurs activités de loisirs? Existe-t-il des différences significatives d'habitudes de voyages et de loisirs selon les modes de vie et les caractéristiques socio-demographiques des producteurs agricoles québécois?

Dans le cadre d'une recherche commanditée ${ }^{[1]}$ par le journal hebdomadaire Terre de Chez Nous de l'Union des producteurs agricoles (UPA) et réalisée par le Centre de recherche en gestion (CRG) de I'UOAM, des données recueillies ont permis de connaître un peu plus les modes de vie et les habitudes de consommation des producteurs agricoles québécois. Un volet exploratoire de cette recherche était orienté vers l'étude de certaines habitudes de voyages et de loisirs.

\section{Méthode de la recherche}

Un échantillon aléatoire proportionnel stratifié par région de cinq mille producteurs agricoles a eté tiré des dossiers informatiques de I'Union des producteurs agricoles. Le questionnaire, accompagné d'une lettre explicative avec entête du Centre de recherche en gestion de I'UOAM et d'une enveloppe pré-affranchie, a êté mis à la poste à la fin d'août 1984. Pour augmenter le taux de réponse, un stylobille était inclus dans l'envoi; et une lettre de rappel fut envoyée deux semaines plus tard. Un total de 2107 questionnaires complétés furent analysés soit un taux de réponse de $42,1 \%$. Le taux de réponse a été plus élevé que prévu considerant que la collecte de données a été faite a la fin de l'été, période oủ la production agricole est la plus active. Nous expliquons ce haut taux de réponse par la méthodologie de l'envoi, l'endossement par un centre de recherche universitaire et la forte appartenance des producteurs agricoles à leur profession.

\footnotetext{
- Pierre Filiatrault est professeur au Departement des sciences administratives de PUOAM et Hans Laroche est diplômể en administration de rUOAM.
}

\section{Le profil des répondants}

Le taux de réponse par question a été relativement élevé (tableau 1). Les répondants étaient en majorité de sexe masculin et de langue française. Un grand nombre $(60,0 \%)$ avaient moins de 45 ans et avaient complété des études primaires et secondaires $(79,1 \%)$. La distribution du nombre d'enfants demeurant à la maison est assez unifrome et les rêpondants provenaient en majorité de la région de Montréal $(38,9 \%)$ et de la région de Québec $(27.1 \%)$. Le revenu familial annuel est inférieur à 20,000 \$ pour $43.0 \%$ des répon= dants. De plus, $38,9 \%$ des répondants avaient des revenus autres que les ventes de produits agricoles et $23,9 \%$ des conjoints travaillaient à l'extérieur. Les données dans cette partie du questionnaire étant surtout nominales et considérant les objectifs de la recherche, I'analyse bivariée retenue fut le Khi carré.

\section{Habitudes de voyages}

Au cours des deux dernières années, $55,0 \%$ des répondants avaient fait un vovage. Parmi ceux qui avaient une seule destination lors de leur dernier voyage de vacances, $57,9 \%$ ont fait ce vovage au Québec. Ces résultats, pour des raisons méthodologiques, ne peuvent être comparés directement aux résultats des enquêtes sur les voyages des Canadiens ${ }^{(2)}$ et des Québécois ${ }^{|3|}$ et les voyages d'agré. ment des Canadiens ${ }^{14}$, mais les résultats sont conformes aux tendances. Les producteurs agricoles québécois voyagaient un peu moins que les autres Québécois et meme un peu moins que les producteurs agricoles canadiens. Cependant, ceux qui voyageraient vont plus souvent à lextérieur que les autres Québécois.

Des différences significatives ont été identifiées dans la propension à voyager des producteurs agricoles québécois par rapport à certaines variables socio-démographiques (tableau 2 en page 28). La propension à voyager est plus forte chez les producteurs agricoles dont les niveaux de scolarité et de revenus sont plus élevés. II $n^{\prime} y$ a pas de différences significatives quant à l'ăge, au nombre d'enfants et à la région, mais les jeunes producteurs ont plus tendance à voyager que leurs aînés.
Des differences très significatives ont été toutefois identifiées par rapport aux variables de modes de vie retenus. Les variables de modes de vie tentent de mieux faire comprendre les habitudes et comportements des consommateurs à partir de la connaissance, entre autres, de leurs activités. Les variables retenues dans cette étude étaient surtout orientées vers les activités qui expliquent le revenu familial total à savoir le nombre de mois actifs comme producteur agricole, la provenance d'autres revenus que les revenus agricoles ot le travail du conjoint a l'extérieur. Les répondants qui ne sont pas actifs douze mois par année, qui ont des revenus autres que ceux des ventes de produits agricoles et/ou dont le conjoint travaille à l'extérieur, voyagent plus (p. .001, pour chacune de ces trois variables).

Les habitudes de voyages au Québec et à l'extérieur du Québec different significativement par rapport à plusieurs variables socio-démographiques et de modes de vie. Les producteurs agricoles dont le niveau de scolarité est plus êlevê, dont le nombre d'enfants est moins élevé et qui proviennent de la grande région de Montréal, ont plus tendance à voyager à l'extérieur du Québec. II n'y a pas de différence quant ả l'âge et au revenu. D'autre part, les répondants qui sont actifs en tant que producteurs agricoles moins de 10 mois par année et dont le conjoint travaille á l'extérieur, voyagent aussi plus hors du Québec que les autres producteurs agricoles québécois; mais, il n'y a pas de différence pour ceux qui ont des revenus autres que les ventes de produits agricoles.

\section{Habitudes de loisirs}

La moitié des répondants disent consacrer sept heures et plus par semaine aux loisirs (lecture, télévision, spectacles, etc.) durant l'automne et l'hiver alors que $36.3 \%$ des reppondans le font durant le printemps et l'été. Les producteurs agricoles québécois lisent beaucoup. La très grande majorițé des producteurs agricoles connaissent l'existence des principaux hebdomadaires et mensuels professionnels, y sont abonnés (de 80 à 


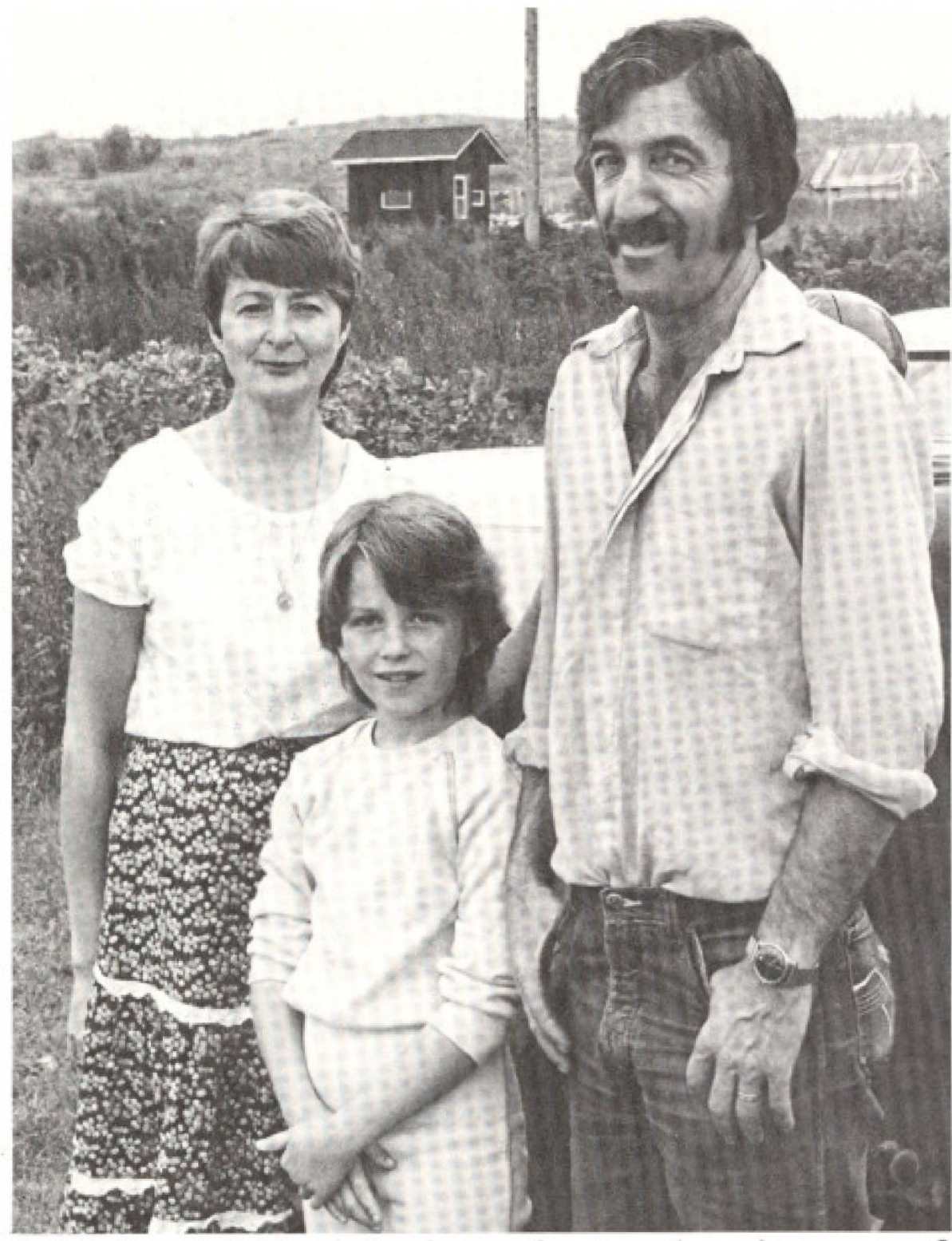

Quelle est la propension des producteurs à voyager durant leurs vacances? Quelles sont leurs destinations? Quelles sont leurs activités de loisirs?

$90 \%$ ) et lisent $50 \%$ et plus de chaque édition de ces publications. Ils considerent que l'information agricole obtenue de ces média est utile $(65,3 \%)$ et plutôt utile $(30,7 \%)$; une évaluation beaucoup plus positive que celle des autres sources d'information. De plus, $76,9 \%$ des répondants ont acheté au moins un livre au cours de la dernière année et $46,2 \%$ ont acheté cinq livres et plus: ils lisent aussi des quotidiens, des hebdomadaires et des revues et magazines mensuels.

Les répondants regardent la télévision surtout durant l'automne et l'hiver; $87,0 \%$ la regardent deux heures et plus par jour durant la semaine, et $85.0 \%$ durant la fin de semaine. Les heures d'écoute chutent durant le printemps et l'été alors que $57,1 \%$ la regardent une heure et moins par jour durant la semaine et $47,4 \%$ durant la fin de semaine. Les producteurs agricoles québécois sont avides d'infor- mation et $73,1 \%$ des répondants regardent régulièrement et $21,6 \%$ regardent á l'occasion les bulletins d'information et les nouvelles à la télévision. Les producteurs agricoles sont également actifs à l'extérieur durant I'hiver, car $49,4 \%$ des répondants possèdent un équipement de ski de fond et $45.8 \%$ possèdent leur motoneige. Enfin, plusieurs habitudes de comportement d'achat et des intentions d'achat de divers produits de consommation ont aussi été étudiés. Nous nous sommes limités dans cet article à l'analyse de l'achat de livres et aux habitudes d'écoute de la télévision.

II existe plusieurs différences significatives dans les comportements d'achat de livres et d'écoute de la télévision (tableau 2). Les répondants qui ont les caractéristi= ques suivantes achètent plus de livres: plus scolairisés, vivant dans la grande région de Montréal, plus actifs comme
Tableau 1

Le profil des rêpondants

SEXE

masculin

fêminin

$\mathrm{n}=1992$

$75.5 \%$

$24.5 \%$

ĀGE

34 ans et moins

$100.0 \%$

35 dे 44 àns

$31.2 \%$

45 aे 54 ans

28.8

55 ans et plus

27.4

$n=2048$

$100.0 \%$

NOMBRE D'ENFANTS

DEMEURANT A LA MAISON

aucur

un

deux

trois

quatre

cing et plus

$\mathrm{n}=2064$

$19.7 \%$

$17.6 \%$

$28.1 \%$

19.5

9.9

5.1

$100.0 \%$

REVENU FAMILIAL

ANNUEL TOTAL

moins de $\$ 10,000$

$\$ 10,000$ a 19,999

$11.4 \%$

31,6

$\$ 20,000$ a 29,999

22.0

$\$ 30,000$ a 39,999

12.5

$\$ 40,000$ a 59,999

11.6

$\$ 60,000$ et plus

$n=2003$

$100.0 \%$

\section{LANGUE MATERNELLE}

francais

anglais et autres

$n=2069$

$97.3 \%$

$2.7 \%$

$100.0 \%$

\section{SCOLARITE}

élémentaire

secondaire

51.9

classique ou collégial

12.4

universitaire

7.2

autres

1.3

$\mathrm{n}=2035$

$100.0 \%$

REGIONS

Bas St-Laurent, Saguenay

Còte-Nord

Québec

Trois-Rivières

12.3

Estrie

7.4

Montréal

38.9

Outaouais Abitibi

6.2

$\mathrm{n}=1999$

$100.0 \%$

REVENUS AUTRES QUE VENTES DE PRODUITS AGRICOLES

oui

$38.9 \%$

non

$n=2068$

TRAVAIL DU CONJOINT A

L'EXTERIEUR

ouit

$23.9 \%$

non

$n=2004$

$76.1 \%$

NOMBRE DE MOIS ACTIFS EN

TANT QUE PRODUCTEURS

AGRICOLES

6.9 mois

10-12 mois

$n=2005$ 
Analyse bivariée (KHI carrél* d'habitudes de voyages et de loisirs de producteurs agricoles québécois par des variables socio-démographiques et de modes de vie

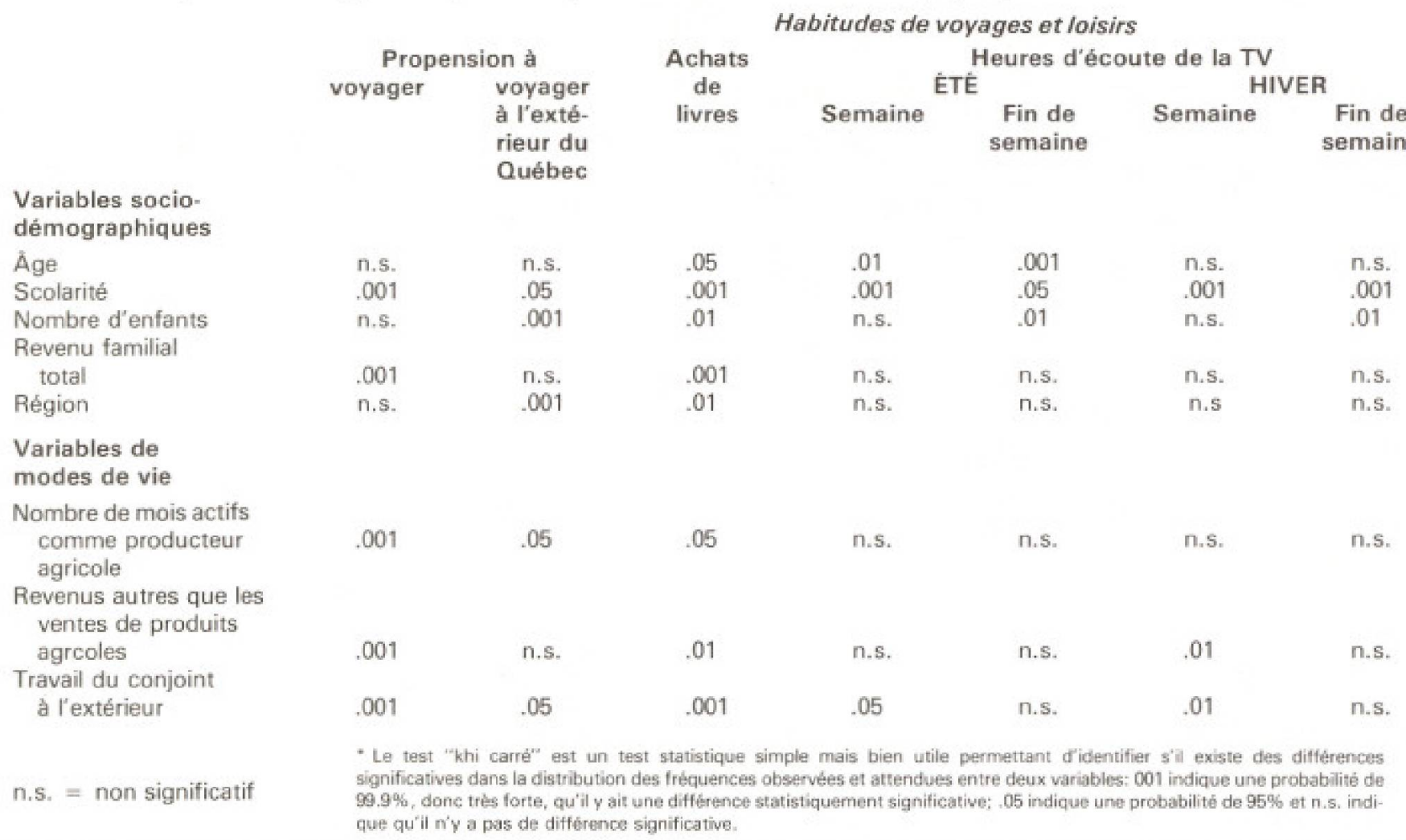

producteurs agricoles, ayant des revenus autres que les ventes des produits agricoles et dont le conjoint travaille à l'extérieur. Les répondants quil sont plus àgés, dont le revenu familial est inférieur à 10,000 \$ ou supérieur à $60,000 \$$ achètent moins de livres.

II n'existe aucune différence significative dans le nombre d'heures d'écoute de la télévision tant durant la semaine que durant la fin de semaine (hiver ou étél par rapport à la région, au revenu familial et au temps actif comme producteur agricole. D'autre part, peu importe le temps de la semaine ou la saison, les répondants plus scolarisés consacrent moins de temps à l'écoute de la télévision. Les personnes plus agées regardent plus la TV durant le printemps et l'èté que les jeunes, mais il n'y a pas de différence durant l'hiver. II existe des différences d'habitudes d'écoute entre la semaine et la fin de semaine dépendant du nombre d'enfants, et si le conjoint travaille à l'extérieur. Enfin, il n'y a pas de différence par rapport au revenu provenant de sources autres que la vente des produits agricoles sauf durant la semaine en hiver alors que les répondants qui ont des revenus provenant d'autres sources regardent moins la télévision.

Les habitudes de voyages des agriculteurs * agricoles québécois ont ensuite été analysées par rapport à leurs habitudes de loisirs. Les producteurs agricoles qui sont allés en woyage au cours des deux dernières années ont acheté plus de livres (p .001) et consacrent plus de temps aux loisirs en automne et en hiver $(p \quad .001)$ et au printemps et en été (p .01) que les autres.

\section{Conclusion}

Dans cette recherche exploratoire sur les habitudes de voyages et de loisirs des producteurs agricoles québécois, nous avons dans un premier temps traçé un profil de ce milieu économique dont on connaît peu le comportement de consommation. Nous avons ensuite étudié la propension à voyager tant au Québec et à l'extërieur du Québec ainsi que certaines habitudes de loisirs, en particulier l'achat de livres et l'écoute de la télévision. Les résultats sur la propension à voyager hors Québec. l'achat de livres, l'achat de diverses publications et le type d'emissions écoutées des producteurs agricoles sont intéressants. Ces résultats ont aussi retenu l'attention des professionnels du milieu agricole auxquels ils ont été présentés, mais la présente étude ne faisait qu'aborder le sujetII existe un besoin évident de poursuivre des recherches en ce sens, permettant de mieux comprendre les habitudes et comportement de consommation des producteurs agricoles québécois, particulièrement en tourisme et loisirs.

\section{Bibliographie}

FILIATRAULT, Pierre et LAROCHE, Hans Étude sur les habitudes et les preferences des lecteurs de publications agricoles du Quebbec. Centre de recherche en gestion. Universite du Quebuc a Montréal, 1984

Stanistique Canada, catalogue 87 504, Enquête sut les vovages des Canadiens. Canadiens vơya. goant au Canada en 1982. Ministere des Approwi sionnemwents et Services Canada, mars 1984.

Gouvemement du Ouebec. Les voyages de plus de 24 heures des Cuebecois au Quebec et a l'extérieur du Québec en 1982. volume 1. Ministète du Touriame, acout 1984

Tourisme Canada, Les intentions de vovages des Canadiens pour les 3 premiers trimestres de 1961 A 1964. Enquele du Conlerence Boand du Canada sur les intentions d'achall des consommateurs, 1984. 